\title{
Posterior Scleritis: a Clinical and Histological Survey
}

\author{
C. M. CALThORPE, P. G. WATSON, A. C. E. McCARTNEY \\ London
}

\begin{abstract}
Summary
The clinical course of 47 patients with posterior scleritis is reviewed. Though clinical presentation varied widely, $73 \%$ of the patients presented with a visual acuity of $6 / 18$ or less. Because the posterior scleritis was not always associated with pain or with anterior scleritis, the diagnosis was often not considered when the patient was first seen. The most common findings in the fundus were disc swelling, retinal detachment, and macular oedema and the most useful investigation was B scan ultrasound. No common aetiology was found, although $60 \%$ had a systemic disorder which was accompanied by a vasculitis. Those who were diagnosed and treated with the minimum delay had the most satisfactory visual outcome. However, there appears to be a group of patients with no underlying systemic disease who fail to respond to intensive therapy, and lose vision. A new sub-group of West Indians with the disease is described. The histopathology of 7 cases confirmed the presence of scleral vasculitis of the vessels in and around the sclera in all the specimens. Other significant findings include inflammatory swelling and focal loss of pigment epithelium together with choroidal vascular closure. This could account for the fluorescein angiographic findings.
\end{abstract}

Posterior scleritis is an inflammatory disorder involving the sclera and episclera of the posterior segment of the eye. It may be associated with anterior scleritis, and may spread to involve muscle and other orbital tissue, or to involve choroid and retina ${ }^{1,2,3}$. The clinical presentation is very variable as it depends on the exact site of the inflammation. This makes the diagnosis difficult. ${ }^{4,5}$ Later in the course of the disease, complications may further obscure the primary condition. ${ }^{6}$ Early diagnosis and initiation of appropriate therapy are essential because delay in treatment inevitably leads to some loss of vision. The presentation and clinical course of a series of 47 patients are described.

\section{Materials and Methods}

A retrospective analysis has been made of 64 eyes of 47 patients with posterior scleritis. Forty one of these were examined in the Scleritis Clinic at Moorfields Eye Hospital and six patients came to our notice through the Pathology Department where their enucleation specimens were examined.

\section{Results}

Sex, Race, Age

Of the 47 patients studied, 28 were female and 19 were male. Forty two were caucasian and the remaining 5 were of West Indian origin. Their ages at presentation ranged from 12 to 87 years. The mean age of all patients was 49 years. The mean age of the caucasians was 57 years; that of the West Indians was 19 years. The mean age of the females, 54 years, was higher than that of the males, 42 years. However, the average age of the males was weighted by 4 young West Indian patients.

\section{Symptoms}

The symptoms on presentation are given in Table I. Forty three patients (91\%) complained of pain of some degree. Of these, only 5 described severe, intolerable pain, 
which prevented sleep. Three patients noted an increase in their pain on ocular movement. Six described radiation of pain to surrounding structures, such as the brow and the jaw.

Table I Symptoms on presentation

\begin{tabular}{lcc}
\hline Symptom & $\begin{array}{c}\text { Number of } \\
\text { patients }\end{array}$ & $\%$ patients \\
\hline Pain & 43 & 91.48 \\
Poor Vision & 38 & 80.85 \\
Red Eyes & 36 & 76.59 \\
Lid Swelling & 9 & 19.14 \\
Photophobia & 4 & 8.51 \\
Diplopia & 4 & 8.51 \\
Joint Pain & 3 & 6.38 \\
Skin Ulcers & 2 & 4.25 \\
\hline
\end{tabular}

Table II Initial site of scleritis

\begin{tabular}{rll}
\hline \multicolumn{2}{l}{ Number of patients (\%) } & Initial site of scleritis \\
\hline 23 & $(48.94)$ & Anterior + Posterior \\
9 & $(19.15)$ & Posterior \\
15 & $(31.91)$ & Anterior \\
\hline
\end{tabular}

Table III Visual acuity at presentation of posterior scleritis

\begin{tabular}{cc}
\hline Visual Acuity & Number of Eyes \\
\hline N.P.L. & 2 \\
P.L.-C.F. & 21 \\
6/60-6/36 & 12 \\
$6 / 24-6 / 18$ & 12 \\
$6 / 12-6 / 9$ & 13 \\
$>6 / 9$ & 4 \\
\hline
\end{tabular}

Table IV External manifestations

\begin{tabular}{lcc}
\hline & No. of eyes & $\%$ eyes \\
\hline $\begin{array}{l}\text { Conjunctival } \\
\text { chemosis }\end{array}$ & 33 & 51.56 \\
Lid swelling & 12 & 18.75 \\
$\begin{array}{l}\text { Impaired ocular } \\
\text { movement }\end{array}$ & 12 & 18.75 \\
Proptosis & 9 & 14.06 \\
Globe tenderness & 6 & 9.37 \\
\hline
\end{tabular}

Thirty six patients $(76 \%)$, noted a red eye initially. Two other patients developed a red eye later in the course of the disease. Of these, 34 had anterior scleritis and 19 had anterior uveitis. Eighteen patients had both anterior uveitis and anterior scleritis. One patient had neither.

\section{Signs}

Site of the inflammation. Five of the 9 presenting with posterior disease alone did not develop anterior disease during the follow-up period (Table II). Of those presenting with anterior disease alone 14/15 developed posterior disease at a mean interval of 1.75 years. (Range immediately to 7 years). The interval in the remaining patient was uncertain. Twelve patients presented with bilateral scleritis, 10 with bilateral posterior scleritis. At the end of the follow-up period, 24 patients had developed bilateral scleritis: 17 of these had bilateral posterior scleritis.

Visual acuity. A profound loss of visual acuity (6/36 or less) was evident in $54 \%$ of eyes. (Table III).

Some external manifestations are listed in Table IV. The maximum degree of proptosis noted was $5 \mathrm{~mm}$. In 3 eyes, the degree of proptosis was not measured. Three patients had impaired ocular movement without an associated proptosis. No ocular nerve palsy was found.

\section{Uveitis}

Of 64 eyes, 45 (70\%) had uveitis. Anterior uveitis was present in 35 eyes, posterior uveitis in 33 eyes, and both anterior and posterior uveitis in 23 eyes.

\section{Shallowing of the anterior chamber}

Shallowing was noted in 9 eyes, although it was not actually measured. Of these, ring choroidal detachment was noted in four.

\section{Glaucoma}

Eleven eyes had raised intra-ocular pressure on presentation. One had angle closure secondary to choroidal detachment. Five had anterior uveitis, and seven had anterior scleritis involving the limbus. Two had both anterior scleritis involving the limbus and anterior uveitis. 
Table V Fundal signs

\begin{tabular}{lcc}
\hline Sign & No. of eyes & $\%$ eyes \\
\hline Optic nerve swelling & 29 & 45.31 \\
Serous retinal detachment & 25 & 39.06 \\
Macular oedema & 17 & 26.56 \\
Subretinal mass & 11 & 17.18 \\
Ring choroidal $\quad$ & 9 & 14.06 \\
$\quad$ detachment & & 12.50 \\
Intra-retinal deposits & 8 & 10.93 \\
Choroidal folds & 7 & 7.81 \\
Pigment epithelial detachment & 5 & 6.25 \\
Subretinal discolouration & 4 & \\
\hline
\end{tabular}

\section{Fundal signs (Table $\mathrm{V}$ ).}

The most common signs were optic nerve swelling, (Fig. 1), serous detachment of the retina (Fig. 2), and macular oedema. Choroidal folds were noted at the posterior pole in 7 eyes. In addition to those with macular oedema, a further 5 eyes were noted to have areas of peripheral retinal oedema, unrelated to clinically obvious subretinal masses. Retinal vasculitis was not noted clinically in any of these patients. However, it was demonstrated on fluorescein angiography in one patient. Pigment epithelial elevations were situated at the posterior pole. Intraretinal cream or white lesions resembling hard exudates were described in 8 eyes. They were not reported in relation to subretinal masses, or retinal oedema. They occurred both at the posterior pole and in the peripheral retina. Four patients had scattered, flat, midperipheral, orange, yellow or pale mottling, at the level of the pigment epithelium, unrelated to obvious subretinal masses. Optic neuropathy was the feature which was common to the group of young, male, West Indian patients but no other combination of clinical signs could be associated with any particular grouping of patients, based on age, sex or associated disease. Eyes commonly had more than one fundal manifestation. Of the 17 with macular oedema, 11 had optic neuropathy, and three a subretinal mass in addition. Of the 29 eyes with optic neuropathy, seven also had retinal detachment. Except for one patient, all those with ring choroidal detachment also had serous retinal detachment.

\section{Ocular investigation}

Fluorescein angiography was carried out on 17 patients. Although uveitis and vitreous haze often rendered the view indistinct, the investigation was found to be useful in distinguishing central serous retinopathy and serous retinal detachment associated with posterior scleritis. (Fig 3).

B-scan ultrasonography was carried out on 20 patients. This test has become useful in recent years when improved techniques have allowed the reliable demonstration of thickened posterior ocular coats. The characteristic signs are illustrated in Figure 4. These include thickening of the sclera and choroid, and an echo free region between the posterior border of the sclera and Tenon's capsule, indicating retrobulbar oedema.

Computerised axial tomography was carried out on 4 patients. However, it was helpful in making a diagnosis in only one.

\section{Associated Systemic Disease}

Sixteen patients $(34 \%)$ were found to have an associated systemic condition (Table VI). The most common was rheumatoid arthritis, which was present in 12 patients. Eleven patients had more than one associated condition. Apart from those listed, 2 patients had thyroid antibodies, and a further 3 had gastric parietal cell antibodies. None of these patients had overt clinical disease affecting these organs. In one patient who was suspected clinically of having Wegener's Granulomatosis several biopsies of sinus, lung, and skin tissue revealed a non-specific arteritis. Three patients died during the fol- 
low-up period: one of myocardial infarction associated with polyarteritis, the second of renal failure, also associated with polyarteritis, and the third of a perforated peptic ulcer secondary to steroid therapy. They all died within 4 years of the onset of their scleral disease.

Table VI Associated systemic disease

Disease

Number of patients

\begin{tabular}{lr}
\hline Rheumatoid Arthritis & 12 \\
Multiple Systemic Auto-immune & 5 \\
$\quad$ Disease & 4 \\
Systemic Arteritis & 1 \\
Beçhets Disease & 1 \\
Myasthenia Gravis & 1 \\
Gout & 1 \\
Hashimotos Thyroiditis & 1 \\
Idiopathic Thrombocytopenic & \\
$\quad$ Purpura & 2 \\
\hline
\end{tabular}

Table VII Systemic Investigation

Haematological screen

Immune screen

Serum uric acid

V.D.R.L., T.P.H.A.

Chest X Ray

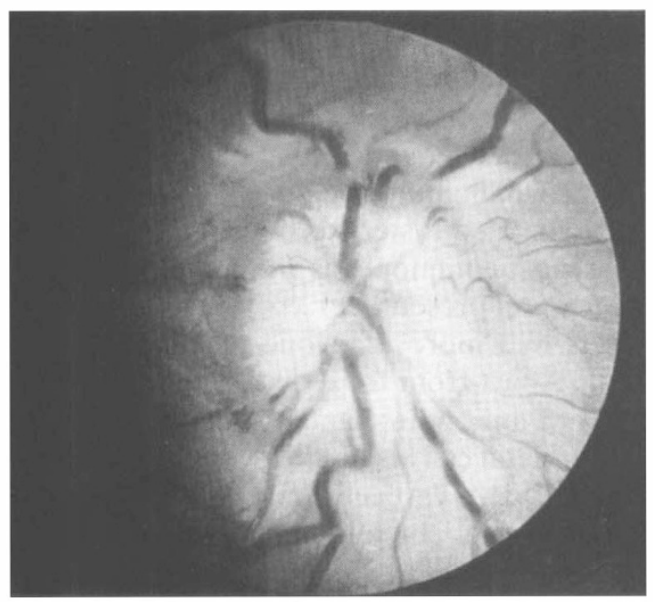

Fig. 1. Swollen optic nerve in a patient who presented with anterior scleritis, pain and deteriorating vision. The diagnosis was confirmed by ultrasound.
Systemic Investigation

All patients underwent those investigations listed in Table VII. Further tests were carried out if indicated on clinical assessment. Laboratory testing, however, did not reveal any systemic disease unsuspected after history taking and clinical examination.

\section{Follow-up}

The mean follow-up period was 4.90 years. The range was 12 months to 19 years. A follow-up of 2 years or more was obtained in $82 \%$ of patients. Posterior scleritis is often a recurrent or chronic condition (Table VIII). Twenty patients suffered recurrent episodes. Of these, 8 remained quiescent between episodes only on maintenance therapy.

Table VIII Activity of the disease

\begin{tabular}{cl}
\hline Number of patients & Number of episodes \\
\hline 18 & 1 \\
9 & 2 \\
6 & 3 \\
3 & 4 \\
1 & 7 \\
1 & 8 \\
9 & Never became quiescent \\
\hline
\end{tabular}

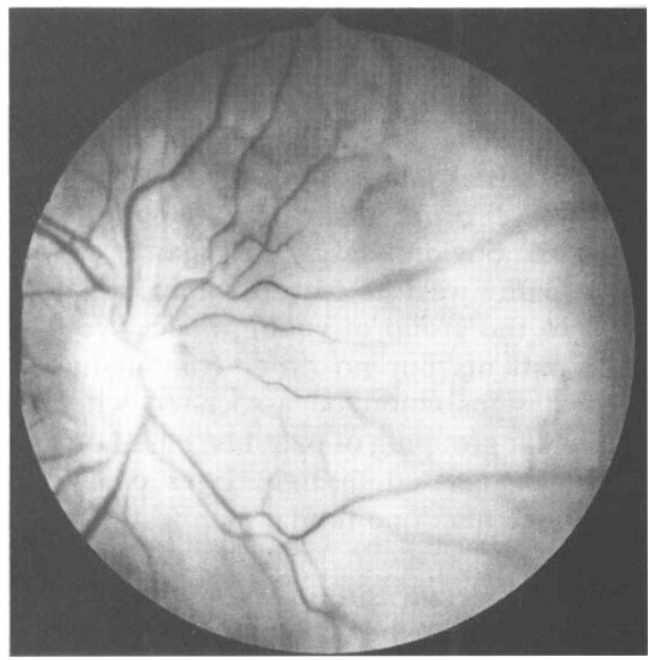

Fig. 2. Serous detachment of the retina at the posterior pole. The diagnosis was confirmed by ultrasound and CT scan. There was no anterior scleritis detected at any stage. 


\section{Treatment}

In the Scleritis Clinic at Moorfields Eye Hospital all patients initially receive a non-steroidal anti-inflammatory (N.S.A.I.) agent, except those with anterior necrotising scleritis, those requiring systemic steroid for the other eye, a previous episode, or a related systemic condition. If steroid is required, Prednisolone $80-120 \mathrm{mg}$ is prescribed daily for 3 days, with a rapidly reducing dose over the following 2 weeks. Those patients not responding to this regime receive an intravenous pulse of between $500 \mathrm{mg}$ and 1 gram of methylprednisolone. Cyclophosphamide $500 \mathrm{mg}$ is given intravenously in addition if the patient is already taking systemic steroid or there are high levels of immune complexes. However, not all patients in this series were treated according to this protocol. Twenty nine patients were managed initially, for varying periods, and six managed wholly, at other centres. The mode of treatment of the entire series of patients, and its duration are shown in Table IX.

Of the six patients who responded to N.S.A.I. agents, none had an associated systemic disease and their scleritis improved within 2 weeks of starting treatment. A seventh patient was only given N.S.A.I agents. He never settled was not given any other treatment, and the eye was enucleated before a diagnosis was made. This patient had rheumatoid arthritis, and colitis.

Of those treated with systemic steroid with or without N.S.A.I. agents, (29 patients), 5 failed to settle. The disease remained quiesant in one patient on maintenance therapy

Table IX Mode/duration of treatment

\begin{tabular}{lccc}
\hline & N.S.A.I. & $\begin{array}{c}\text { Systemic Steroid }+ \text { /- } \\
\text { N.S.A.I. }\end{array}$ & $\begin{array}{c}\text { Systemic Steroid }+ \\
\text { otherimmuno } \\
\text { suppressive agent }\end{array}$ \\
\hline \% Patients & 14.90 & 61.70 & 23.40 \\
$\begin{array}{l}\text { Mean duration } \\
\text { treatment } \\
\text { (months) }\end{array}$ & 1.0 & 14.34 & 24 \\
\hline
\end{tabular}
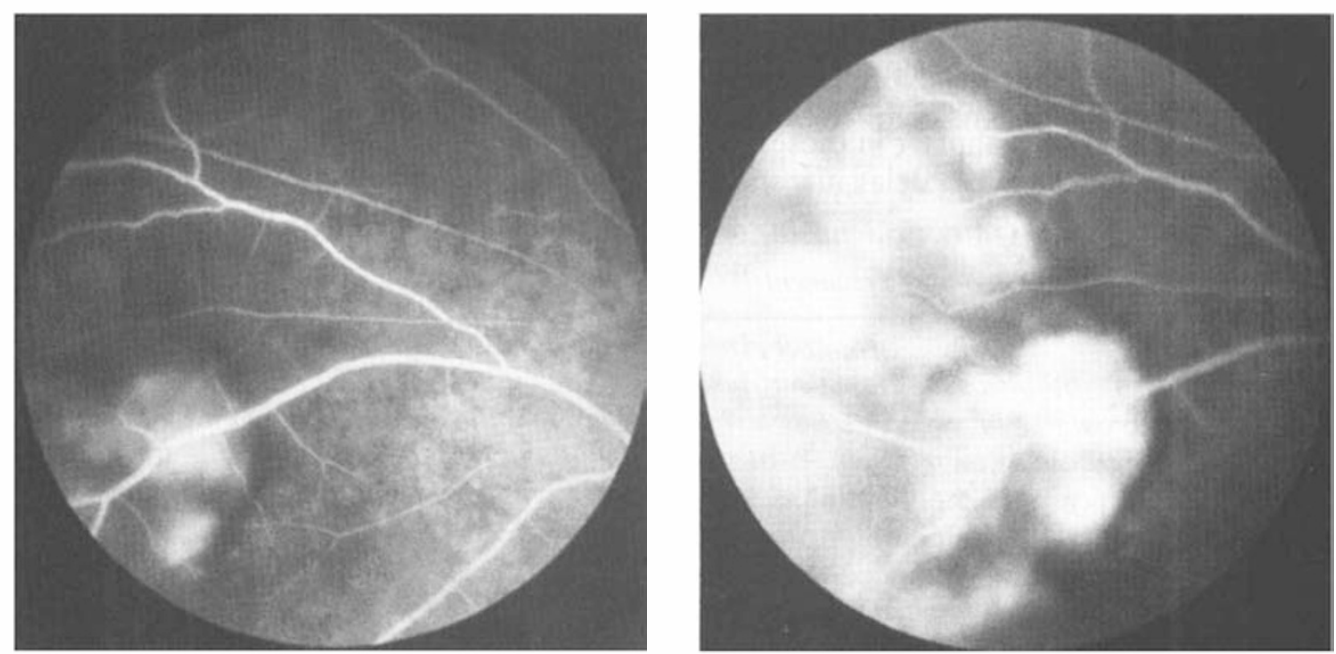

Fig. 3a,b. A fluorescein angiogram of an area of scleritis with overlying retinal detachment, showing choroidal hypofluorescence (a), and patchy fluorescence in a later frame (b) due to leakage of fluorescein into the subneuroretinal space. 
alone. Of this group, $36 \%$ had an associated systemic disease. Eleven patients required either cyclophosphamide or azathioprine in addition to systemic steroid. In 7 of the 11 patients this was administered by pulsed therapy. In spite of this three failed to settle. Fifty-four per cent of these patients had an associated systemic disease. One patient, whose retinal-detachment failed to settle after 11 months of systemic steroid and cyclophosphamide therapy, underwent surgical exploration. A grossly thickened sclera was found, constricting a vortex vein. A window of sclera was removed, relieving the constriction. The retina rapidly re-attached postoperatively and has remained so for the follow-up period of 15 months.

Forty patients were treated on systemic steroid for a mean period of 17 months and eleven required either azathioprine or cyclophosphamide in addition. The drugs used added considerably to morbidity. Sixteen patients $(34 \%)$ developed one or more systemic complications of treatment. These are listed in Table X. One patient died of a perforated peptic ulcer induced by systemic steroid, which was required for both her rheumatoid arthritis and scleral disease.

\section{Visual Outcome}

Patients are divided into 4 groups according to their visual outcome (Table XI). Over $50 \%$ of eyes had a visual acuity of $6 / 9$ or better at the end of the follow-up period. The mean interval from the onset of symptoms to making the diagnosis is shorter in those with a better visual outcome. The delay in institut-

Table X Systemic complications of Treatment

\begin{tabular}{lc}
\hline Complication & $\begin{array}{c}\text { Number of } \\
\text { patients }\end{array}$ \\
\hline High blood sugar & 5 \\
Mental changes & 4 \\
Peptic ulceration & 2 \\
Gastritis & 2 \\
Anaemia & 2 \\
Myopathy & 2 \\
Hypertension & 1 \\
Obesity & 1 \\
Steroid acne & 1 \\
\hline
\end{tabular}

ing adequate treatment affected the visual outcome. The most common cause being the effect of prolonged inflammation, and nerve fibre layer degeneration associated with optic neuropathy. Fourteen eyes had a visual

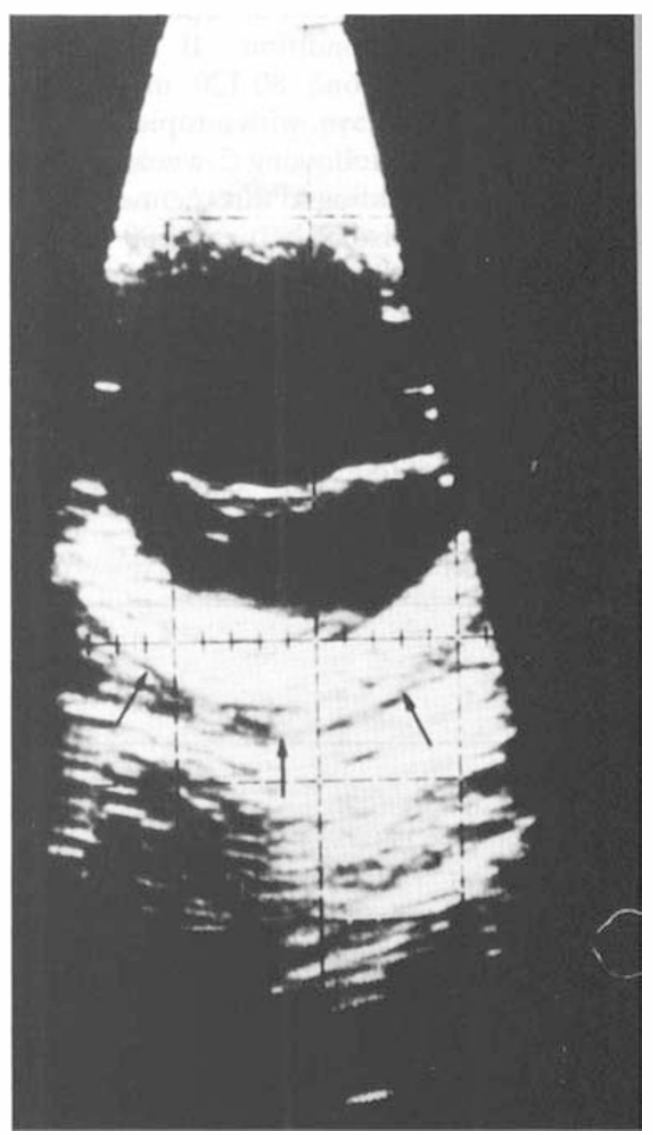

Fig. 4. B-scan ultrasound in eyes with posterior scleritis. Serous elevation of the retina, thickened posterior ocular coats, and an echo-free region between the posterior border of the sclera and Tenon's capsule (arrows).

Table XI Visual acuity related to mean interval to diagnosis

\begin{tabular}{ccc}
\hline $\begin{array}{c}\text { Number of eyes } \\
(\%)\end{array}$ & Visual Outcome & $\begin{array}{c}\text { Mean interval } \\
\text { to diagnosis }\end{array}$ \\
\hline $33(51.56)$ & $6 / 9-6 / 4$ & 3.64 \\
$8(12.50)$ & $6 / 24-6 / 12$ & 6.75 \\
$9(14.06)$ & C.F.-6/36 & 8.00 \\
$14(21.88)$ & N.P.L.-H.M. & 10.28 \\
\hline
\end{tabular}


Table XII Visual acuity before and after treatment

\begin{tabular}{lcc}
\hline Visual Acuity & $\begin{array}{c}\text { Before Treatment } \\
\text { Number of eyes (\%) }\end{array}$ & $\begin{array}{c}\text { After Treatment } \\
\text { Number of eyes (\%) }\end{array}$ \\
\hline N.P.L.-H.M. & $7(10.94)$ & $14(21.88)$ \\
C.F. - 6/36 & $28(43.75)$ & $9(14.06)$ \\
6/24- $6 / 12$ & $17(26.56)$ & $8(12.50)$ \\
$6 / 9-6 / 4$ & $12(18.75)$ & $33(51.56)$ \\
\hline
\end{tabular}

acuity ranging from N.P.L. to H.M. at the end of the follow-up period, ( 7 eyes were in this state when first seen) (Table XII). The mean interval from onset of symptoms to the establishment of the diagnosis and institution of treatment was longer in this group (Table XII).

\section{West Indian Patients}

Since this group of patients was considerably younger, their features will be considered separately, in addition to being included in the main group. Seven eyes of 5 patients presented with posterior scleritis. Four of the 5 patients were male. Their mean age was 19 years. The mean interval to diagnosis was 13.5 months. The visual acuity before and after treatment is shown in Table XIII. All had anterior scleritis and all had evidence of optic neuropathy. None had an associated systemic disease. Three patients suffered recurrent episodes of scleritis. Four patients were treated with systemic steroid, and one with a N.S.A.I. agent alone. Though the mean interval to treatment was long, this group responded rapidly to treatment. The longest period of treatment required was 4 months. Five of 7 eyes had a visual acuity of $6 / 12$ or better at the end of the follow-up period. Permanent loss of vision was due to optic atrophy following prolonged optic nerve swelling.

\section{Enucleation}

Eight eyes were enucleated for various reasons (Table XIV). Of these, the diagnosis was made only on histological examination in six. Six of these patients were female. Four had multiple systemic auto-immune disease. The remaining patients were well. The mean interval from onset of the condition to the establishment of the diagnosis was 13
Table XIII Visual acuity of West Indian patients

\begin{tabular}{lll}
\hline Eye & \multicolumn{2}{c}{ Visual Acuity } \\
& Before treatment & After treatment \\
\hline 1 & $\mathrm{CF}$ & $6 / 12$ \\
2 & $\mathrm{NPL}$ & $\mathrm{NPL}$ \\
3 & $\mathrm{CF}$ & $6 / 6$ \\
4 & $6 / 60$ & $6 / 60$ \\
5 & $6 / 60$ & $6 / 6$ \\
6 & $6 / 18$ & $6 / 6$ \\
7 & $6 / 18$ & $6 / 9$ \\
\hline
\end{tabular}

Table XIV

Number of eyes Reason for enucleation

$\begin{array}{ll}5 & \text { Painful Blind Eye } \\ 2 & \text { Suspected Malignancy } \\ 1 & \text { Phthisis Bulbi }\end{array}$

months. Seven patients were treated with systemic steroid. However, 6 of these were not treated according to the protocol in use at present. None responded to treatment, and they suffered prolonged periods of inflammation (mean 42 months) prior to their enucleation.

\section{Pathology}

Material was available for examination in seven cases (Table XV). Two of these, (cases 1 and 3) were scleral biopsies. These eyes were examined in celloidin, thus immunohistochemistry was not possible. The dominant cell types found were chronic inflammatory cells. These were lymphocytes and plasma cells ( 7 cases), macrophages (4 cases), and giant cells ( 2 cases). Acute inflammatory cells were seen in 3 cases but were few in number. Active scleral vasculitis was noted in 
Table XV

\begin{tabular}{ccc}
\hline Case & Systemic Disease & Scleral vasculitis \\
\hline 1 & & + \\
2 & & + \\
3 & & + \\
4 & Rh.A.,Colitis & + \\
5 & M-S.A-I.D. & + \\
6 & M-S.A-I.D. & + \\
7 & & \\
$*$
\end{tabular}

6 cases. Previous vasculitis diagnosed by 'onion skin thickening' was present in another (case 1). Smudging and fragmentation of scleral collagen, with loss of polarisation was seen in 3 cases. Only 1 (case 2) had necrotising scleritis.

The choroid was involved in all enucleated eyes and was thickened with inflammatory infiltrate (Fig. 5a). Choroidal vasculitis was noted in 4 cases, and onion skin thickening with occlusion of vessels in one of these (Fig. 5a). No evidence of choroidal infarction was found.

In 2 cases the retinal pigment epithelium (RPE) was absent focally with inflammation in the surrounding RPE (Fig. 5b). These RPE. lesions were in continuity with underlying choroiditis and scleritis. Retinal vascular cuffing was noted in 4 cases (Fig. 6). However, retinal vasculitis was not found. Subneuroretinal exudate was found in 2 cases, and was related to areas of scleral vasculitis.

\section{Discussion}

Posterior scleritis has many features which are shared with other diseases. Thus it has been confused with angle closure glaucoma, optic neuritis, central serous retinal detachment, pigment epithelial detachment, rhegmatogenous retinal detachment, primary $4,7,8$ or secondary 9 choroidal tumour, orbital inflammatory disease or mass, the uveal effusion syndrome, placoid pigment epitheliopathy, accelerated hypertension, and Harada's syndrome. Although these patients present early because their symptoms are difficult to ignore, the diagnosis is often delayed. This is borne out by this study, in which the mean interval from presentation to diagnosis was over 7 months. The diagnosis of posterior scleritis is aided by a high level of suspicion and confirmed by ultrasonography. Thus posterior scleritis is almost certainly a more common condition than has been previously supposed. 4,6

This series of 47 patients is similar in age and sex distribution to those previously described, ${ }^{5,10,11}$ except for that of Singh et al ${ }^{12}$ eight of whose 9 cases were male.

The most common presenting symptoms of this series were pain and loss of vision. Those with posterior disease alone had either no pain or pain of a mild degree. The pain may almost certainly be ascribed to anterior scleritis, ${ }^{5}$ which was present in $81 \%$ of patients. Other possible causes of pain include Tenonitis, stretching of nerves passing through Tenon's capsule and sclera; ${ }^{4}$ and optic neuritis associated with inflammation of the nerve sheath. ${ }^{12}$

A poor corrected visual acuity of $6 / 18$ or less was noted in $73 \%$ of eyes at presentation. This contrasted with the near normal visual acuity of Singh's patients. ${ }^{12}$

Anterior scleritis was present or had occurred in $81 \%$ of our patients. Evidence of posterior disease should therefore be sought throughout the course of any episode of anterior scleritis, but equally, the absence of anterior inflammation should not preclude the possibility of posterior scleritis. Uveitis was present in $69 \%$ of eyes. This is a considerably higher figure than that seen in anterior scleritis alone. ${ }^{10}$ The presence of uveitis with anterior scleritis, therefore, is a useful indicator of posterior disease.

Only one patient developed glaucoma as a direct result of posterior segment disease, and presented with angle closure glaucoma. This mode of presentation has been documented in the past. ${ }^{13}$ The remaining 11 patients with glaucoma had uveitis or scleritis involving the drainage area, or both. This agrees with the observation of Wilhelmus $e t$ $a l^{14}$ that damage to the trabecular meshwork by uveitis and overlying corneoscleral inflammation is the most common cause of raised intra ocular pressure in scleritis.

A wide variety of fundal signs is seen in posterior scleritis ${ }^{4,5,15,16}$. These include optic 

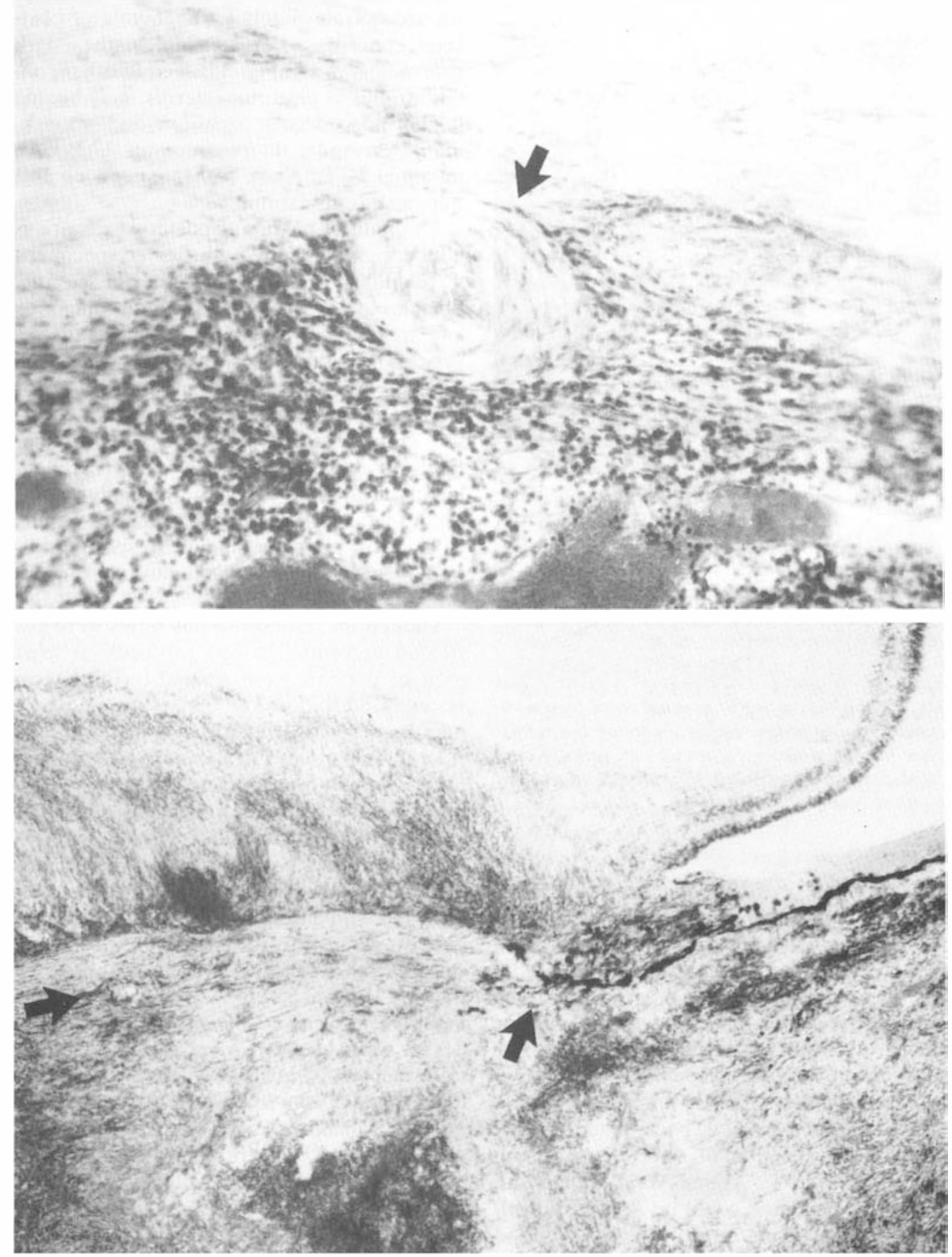

Fig. 5a,b. a. Thickened, inflammed choroid, with obliteration of a small vessel (arrow). X 180, H+E, Celloidin.

b. Active scleral and choroidal inflammation (arrow). Note abrupt cessation of RPE (arrow). The retina (upper left) shows disruption of $\times 180, H+E$, Celloidin. Architecture and infiltration by inflammatory cells. 


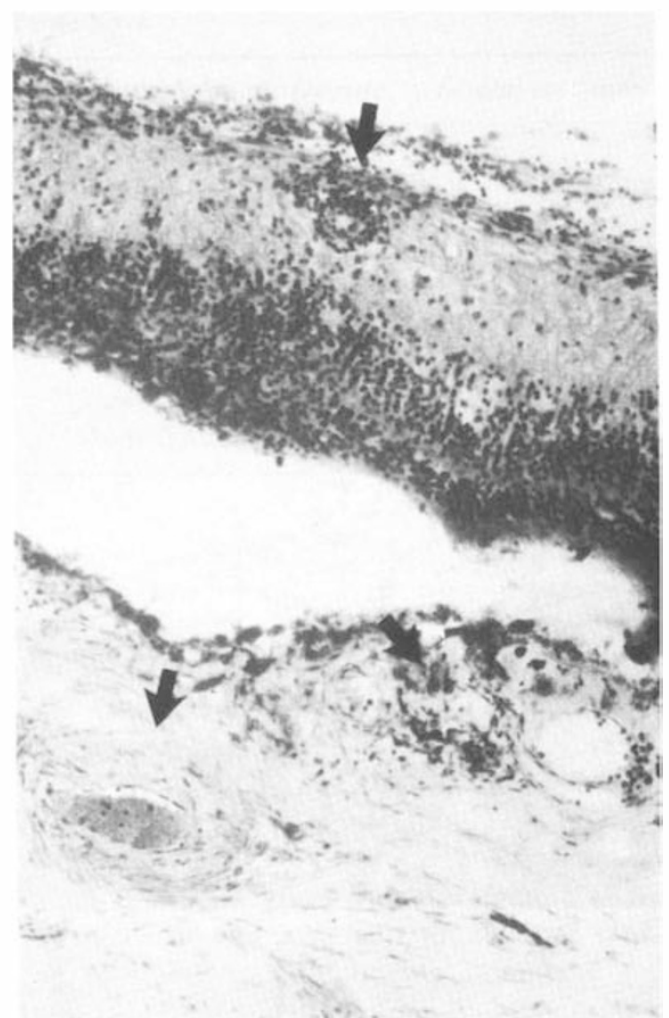

Fig. 6. Marked cuffing of a retinal vessel (arrow) in active chronic inflammation involving the retina. Note thickened choroidal vessel wall (arrow), and focal disturbance of the RPE with incontinence of pigment (arrow). $\times 180, H+E$, Celloidin.

disc swelling, retinal detachment, macular oedema, subretinal mass, choroidal detachment, choroidal folds and pigment epithelial detachment. This presentation should not be overlooked in children. These patients may be pain free and without evidence of anterior scleritis or uveitis. The diagnosis of posterior scleritis should be considered, therefore, in any patient with a fundal lesion, in whom a firm diagnosis has not been made. Apart from the association of optic disc swelling with the group of young West Indians no other manifestation could be associated with any particular subgroup of this series based on age, sex, or associated systemic disease.

Ultrasonography is the most useful investigation since it provides diagnostic signs of posterior scleritis ${ }^{17,18}$ and see Fig. ${ }^{4}$. Unfortu- nately the angiographic features whilst helpful are not absolutely pathognomic of posterior scleritis. For example, the dark choroid, shown in Fig. 3 is seen in other conditions and in posterior scleritis, may be due to choroidal vascular occlusion, and/or masking of choroidal fluorescence by thickened, inflamed RPE, since both are seen on histological examination.

The natural history of posterior scleritis is not benign. Six of the 8 enucleated specimens were undiagnosed cases of posterior scleritis. Furthermore, those cases whose diagnosis and treatment were delayed longest had a very poor visual outcome.

When the interval from onset of posterior scleritis to initiation of treatment was short, the visual outcome was most favourable. Even so 3 patients who were in excellent health and were treated energetically at presentation lost vision permanently. Apart from their poor therapeutic response, initially they were indistinguishable from others.

Though the patients in this series were not treated according to one protocol, at least $85 \%$ of patients were treated on long-term systemic steroid for a mean period of 21 months. The associated morbidity was considerable. Pulsed intravenous steroid and other immunosuppressive therapy were introduced to minimise the side effects of steroids. ${ }^{19,20,21,22}$

Sixteen patients $(34 \%)$ had an associated systemic disease. This is less than that reported with anterior scleritis. ${ }^{10}$ It was these patients who more often required systemic steroid with additional immuno-suppressive therapy and needed longer periods of treatment.

The seven cases that were examined histologically illustrate the wide range of features that are seen in posterior scleritis. Loss of RPE, which has not been noted before was seen in 2 cases. This retrospective examination confirms the previous observation ${ }^{23}$ that chronic inflammatory cells are prevalent and the presence of mast cells is a prominent feature. Increased numbers of mast cells were seen in 3 cases. These cells are thought to initiate the cascade of $\mathrm{T}$ cell-mediated delayed hypersensitivity, and influence B cell 
cycles and the secretion of vasoactive factors. ${ }^{24}$ Only one of the 7 cases was of the necrotising variety. $23,25,26$ Histology, in this instance, shows the typical smudged and fragmented collagen with widespread granulomatous change. Evidence of scleral vasculitis was noted in all 7 cases, confirming that a vasculitis is an underlying feature of scleritis. ${ }^{4}$

We thank Professor A. C. Bird for advice on the interpretation of the fluorescein angiography, Miss $\mathbf{M}$. Restori for the ultrasound studies, and Moorfields Eye Hospital Audiovisual Department.

\section{References}

${ }^{1}$ Chavis RM, Garner A, Wright JE: Inflammatory Orbital pseudotumour. A clinicopathologic study. Arch ophthalmol 1978; 96: 1817-22.

${ }^{2}$ Rootman J, and Nugent R: The classification and management of acute orbital pseudotumours. Ophthalmol 1982; 89: 1040-8

${ }^{3}$ Bertelsen TI: Acute sclerotenonitis and ocular myositis complicated by papillitis, retinal detachment and glaucoma. Acta Ophthalmol 1960; 38: 136-52.

${ }^{4}$ Watson PG: The nature and treatment of scleral inflammation. Trans Ophthalmol Soc UK 1982; 102: 257-81.

${ }^{5}$ Benson WE, Shields JA, Tasman W, Crandall AS: Posterior Scleritis. A cause of diagnostic confusion. Arch Ophthalmol 1979; 97: 1482-6.

${ }^{6}$ Fraunfelder FT and Watson PG: Evaluation of eyes enucleated for scleritis. $\mathrm{Br} \mathrm{J}$ Ophthalmol 1976; 60: 227-30.

${ }^{7}$ Sears ML: Choroidal and retinal detachments associated with scleritis. Am J Ophthalmol 1964; 58, 764-6.

${ }^{8}$ Feldon SE, Singleman J, Albert DM, Smith TR: Clinical manifestations of brawny scleritis. Am J Ophthalmol 1978; 85: 781-7.

${ }^{9}$ Yeo JH, Jakobiec FA, Iwamoto T, Brown R, Harrison W: Metastatic carcinoma masquerading as scleritis. Ophthalmology 1983; 90: $184-94$.

${ }^{10}$ Watson PG and Hayreh SS: Scleritis and episcleritis. Br J Ophthalmol 1976; 60: 163-91.

${ }^{11}$ McGavin DDM, Williamson J, Forrester JV, Foulds WS, et al : Episcleritis and scleritis: a study of their clinical manifestations and association with rheumatoid arthritis. $\mathrm{Br} J$ Ophthalmol 1976; 60: 192-233.

12 Singh G, Guthoff R, Foster CS: Observations on longterm follow-up of posterior scleritis. Am J Ophthalmol 1986; 101: 570-5.

${ }^{13}$ Quinlan MP and Hitchings RA: Angle-closure glaucoma secondary to posterior scleritis. $\mathrm{Br} \mathrm{J}$ Ophthalmol 1978; 62: 330-5.

${ }^{14}$ Wilhelmus KR, Grierson I, Watson PG: Histopathologic and clinical associations of scleritis and glaucoma. Am J Ophthalmol 1981; 91: 697-705.

${ }^{15}$ Cleary PE, Watson PG, McGill JI, Hamilton AM: Visual loss due to posterior segment disease in scleritis. Trans Ophthalmol Soc UK 1975; 95: 297-300.

16 Berger B and Reeser F: Retinal pigment epithelial detachments in posterior scleritis. $A m \mathrm{~J}$ Ophthalmol 1980; 90: 604-6.

${ }^{17}$ Cappaert WE, Purnell EW, Frank KE. Use of B-Sector scan ultrasound in the diagnosis of benign choroidal folds. Am J Ophthalmol 1977; 84: 375-9.

${ }^{18}$ Rochels R and Reis G: Echographie bei skleritis posterior. Klin Mbl Augenheilk 1980; 177: 611-13.

${ }^{19}$ Foster CS: Immunosuppressive therapy for external ocular inflammatory disease. Ophthalmol 1980; 87: 140-9.

${ }^{20}$ Wakefield D, McCluskey P, Penny R. Intravenous pulse Methylprednisolone therapy in severe inflammatory eye disease. Arch Ophthalmol 1986; 104: 847-51.

${ }^{21}$ McCluskey $\mathrm{P}$ and Wakefield D: Intravenous Pulse Methylprednisolone in Scleritis. Arch Ophthalmol 1987; 105: 793-7.

22 Myer PA, Watson PG, Franks W, Dubord P. 'Pulsed' Immunosuppressive Therapy in the treatment of immunologically induced corneal and scleral disease. Eye 1987; 1: 487-95.

${ }^{23}$ Young RD and Watson PG: Microscopical studies of necrotising scleritis. 1. Cellular aspects. Br J Ophthalmol 1984; 6: 770-80.

${ }^{24}$ Askenase PW and Van Loveren H: Delayedtype hypersensitivity: activation of mast cells by antigen specific T-cell factors initiates the cascade of cellular interactions. Immunology Today 1983; 4: 259-64.

25 Young RD and Watson PG: Microscopical studies of necrotising scleritis. 11. Collagen degradation in the scleral stroma. $\mathrm{Br} J$ Ophthalmol 1984; 68: 781-9.

${ }^{26}$ Sevel D: Necrogranulomatous scleritis: clinical and histological features. Am J Ophthalmol 1967; 64: 1125-34. 